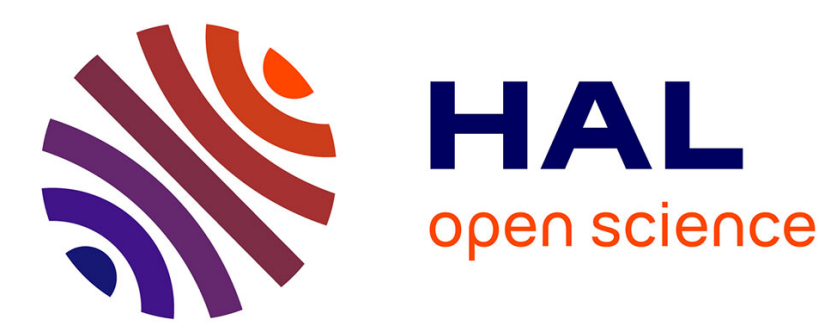

\title{
Certification: création d'un outil de comparaison des différents systèmes existant de par le monde. \\ Patrick Ollivier
}

\section{To cite this version:}

Patrick Ollivier. Certification: création d'un outil de comparaison des différents systèmes existant de par le monde.. Revue forestière française, 2001, 53 (6), pp.653-660. 10.4267/2042/4953 . hal03442641

\author{
HAL Id: hal-03442641 \\ https://hal.science/hal-03442641
}

Submitted on 23 Nov 2021

HAL is a multi-disciplinary open access archive for the deposit and dissemination of scientific research documents, whether they are published or not. The documents may come from teaching and research institutions in France or abroad, or from public or private research centers.
L'archive ouverte pluridisciplinaire HAL, est destinée au dépôt et à la diffusion de documents scientifiques de niveau recherche, publiés ou non, émanant des établissements d'enseignement et de recherche français ou étrangers, des laboratoires publics ou privés. 


\title{
Certification : CRÉATION D'Un OUTIL DE COMPARAISON DES DIFFÉRENTS SYSTÈMES EXISTANT DE PAR LE MONDE
}

\author{
Patrick Ollivier
}

Après une première période de confusion vers le milieu des années 1990 (" qu'est-ce que c'est encore que ce truc-là ? "), puis de rejet (" ça ne sert à rien, nous gérons parfaitement nos forêts »), le concept d'écocertification est entré dans le paysage forestier et économique.

Le terme d'écocertification est d'ailleurs un raccourci, et les puristes, il y a encore peu de temps, expliquaient qu'il fallait parler "de la certification de la durabilité de la gestion forestière" ; mais la paresse naturelle de l'homme et la tendance qui veut que l'on désigne par un mot simple (si ce n'est par une série d'initiales) un ensemble de choses assez complexes, ont fait que le terme d'écocertification s'est finalement imposé.

Au-delà de l'épiphénomène sémantique, la pression des marchés a joué, et sont alors apparus différents systèmes visant, au final, d'une part à rassurer l'acheteur de produits en bois sur la qualité de la gestion des forêts d'où est issu le matériau qu'il va acheter et d'autre part à au moins maintenir sinon améliorer le niveau de cette qualité.

Ces systèmes, qui visent donc tous le même but, ont néanmoins été montés dans des contextes géographiques, écologiques et sociaux différents, parfois en réaction à d'autres, et il est vite apparu que leur prolifération allait sérieusement compliquer la compréhension des gens auxquels ils étaient en fin de compte destinés.

Et comme derrière chacun de ces systèmes existent - il ne faut pas se le cacher - des aspirations économiques et parfois idéologiques, chacun tente évidemment de séduire le maximum de gens. Les arguments, pour ce faire, ont souvent été terriblement primaires, comme les vieilles réclames d'autrefois : "Le système de Certification XYZ est le SEUL système valable, et d'ailleurs il est inutile d'en développer d'autres "; on était alors dans une situation où l'incantation, ou au mieux la conviction subjective, prétendait suffire pour définir des valeurs.

\section{UNE VOLONTÉ DE SE DOTER D’UN OUTIL DE COMPARAISON RATIONNELLE DES SYSTÈMES : LA MATRICE CEPI}

Face à ce manque de rationalisation, et aussi ayant conscience que beaucoup de ceux qui demandent l'écocertification en connaissent en fin de compte mal les arcanes, la Confédération européenne des Industries du Papier (CEPI) a pris l'initiative de construire une matrice de comparaison des différents systèmes existant sur le "marché", et de la mettre à la disposition du public. 
Ce travail s'est appuyé sur deux concepts principaux :

- La matrice ne doit que comparer les différents systèmes recensés, sans porter de jugement : elle laisse donc à chacun le soin de formuler sa propre opinion. En revanche, pour ce faire, elle fournit une "matière" objective et documentée.

- Afin que chaque système soit étudié avec toute l'objectivité nécessaire, et que cette approche soit identique, un groupe d'experts au sein de la CEPI a établi la liste des caractéristiques que "l'honnête homme" s'attend à voir respecter par un système. Cette liste a ensuite été discutée avec les parties tierces, de façon à arriver à une vision aussi complète que possible (cf. encart, p. 655).

À partir de ces principes, la CEPI a fait établir un questionnaire-type qui a été adressé à tous les systèmes recensés. Les réponses ont été dépouillées par un consultant indépendant internationalement reconnu (l'Anglais Rupert Oliver).

Enfin, le même groupe d'experts de la CEPI, à nouveau en relation avec les parties intéressées, a établi la trame d'une matrice de comparaison, qui accompagne cet article.

\section{LA MATRICE DE COMPARAISON CEPI DANS SON ÉTAT ACTUEL}

\section{Constitution de l'information présentée dans la matrice}

Les réponses reçues des différents systèmes ont donc été dépouillées par le consultant de façon à entrer dans la présentation synthétique de la matrice. Toutefois, l'analyse qui en a été faite a d'abord été communiquée aux émetteurs des réponses, ces dernières étant souvent accompagnées de questions complémentaires permettant de mieux établir la synthèse : tous les systèmes comparés par la matrice ont donc eu la possibilité de valider l'information publiée les concernant.

Cependant, comme la publication de la matrice est un phénomène "vivant", qui fait état d'une situation au moment de sa publication, les informations publiées sont identifiées comme relevant de trois cas possibles :

- version préliminaire (Preliminary draft) indique que l'information portée n'est - à ce stade que le seul fait de l'analyse du consultant à partir des réponses brutes faites au questionnaire.

- version amendée (Amended draft) indique que les réponses reçues à travers le questionnaire ont en plus été complétées par des commentaires additionnels envoyés par le système.

- version confirmée (Confirmed) indique que le système a eu connaissance de l'information publiée le concernant, et a eu le temps de la valider.

\section{Structure de la matrice de comparaison}

La matrice décrit les différents systèmes qui ont répondu à son questionnaire. En colonnes sont présentés les critères et indicateurs définis sur la base des onze caractéristiques exposées p. 655 . En lignes sont présentés les systèmes, classés selon qu'ils ont une vocation internationale ou nationale.

- Les systèmes à vocation nationale impliquent le développement de standards adaptés, à travers un processus participatif ; ils n'ont en général pas dimension à aller jusqu'aux questions d'étiquetage ; aussi ces points ne sont pas abordés pour eux dans la matrice.

- Les systèmes à vocation internationale cherchent plutôt à incorporer la reconnaissance de systèmes nationaux et le développement de règles et procédures s'étendant à l'étiquetage.

En revanche, les systèmes à vocation internationale ne sont en général pas impliqués dans le développement de bases détaillées : cette partie-ci ne les concernera donc pas dans la matrice. 


\section{CARACTÉRISTIQUES ATTENDUES D’UN SYSTÈME DE CERTIFICATION}

1. La certification ne doit pas introduire de discrimination entre les différents types de forêts et entre les différents types de propriété forestière.

2. Les organismes certificateurs doivent être indépendants et impartiaux, sans intérêts commerciaux avec les entités à certifier, et doivent disposer d'un personnel compétent et expérimenté sur ces sujets.

3. Les organismes certificateurs doivent être accrédités au niveau national, à travers des méthodes et des organismes internationalement reconnus.

4. La certification doit se faire par rapport à des bases de procédures ou de performance qui soient compatibles avec les principes et critères de gestion durable internationalement reconnus.

5. Ces bases doivent être compatibles avec les politiques forestières nationales et les réglementations afférentes.

6. Les standards et les schémas généraux de la procédure doivent être développés à travers un processus ouvert, associant toutes les parties intéressées et garantissant que les points de vue seront pris en compte de manière équilibrée. Aucun groupe d’intérêt particulier ne doit dominer le processus.

7. La certification doit être transparente. Il doit y avoir des procédures et une documentation claires.

8. La procédure de certification doit être répétable, de façon à ce qu'elle arrive à un même résultat quel que soit l'organisme qui la réalise.

9. Les bases de standards et procédures détaillées doivent être adaptables aux évolutions, et régulièrement revues, de façon à ce qu'elles restent toujours en phase avec les évolutions des connaissances forestières, et les évolutions des aspirations politiques, sociales, économiques et écologiques vis-à-vis de la certification.

10. La certification de la gestion forestière doit demeurer peu coûteuse et commercialement supportable.

11. Si le schéma doit déboucher sur une forme d'étiquetage à modalité unique des produits, il doit s'appuyer sur un audit par tierce partie, pour la chaîne de contrôle des bois.

Enfin, les questions relatives à l'évaluation de la compétence des certificateurs pourront être traitées aussi bien au niveau des systèmes internationaux que nationaux.

\section{Lecture de la matrice de comparaison}

Toutes les réponses reçues à travers des questionnaires et des informations complémentaires envoyés par les systèmes ont été dépouillées par rapport à la liste CEPI des "caractéristiques attendues".

L’information synthétique qui en est tirée est présentée dans le tableau suivant:

\begin{tabular}{|l|l|}
\hline$++\ldots$ & le système satisfait pleinement au critère concerné \\
\hline$+\ldots \ldots$ & le système ne satisfait que partiellement au critère concerné \\
\hline $0 \ldots \ldots$ & le système ne satisfait pas au critère concerné \\
\hline$\ldots \ldots$ & $\begin{array}{l}\text { les réponses ne fournissent pas une information suffisante pour évaluer le système par rapport } \\
\text { au critère concerné }\end{array}$ \\
\hline na $\ldots$ & le critère ne s’applique pas \\
\hline
\end{tabular}


Tableau des principaux indicateurs :

\begin{tabular}{|c|c|c|c|c|c|c|c|c|c|c|c|c|c|c|c|c|}
\hline \multirow[b]{2}{*}{ 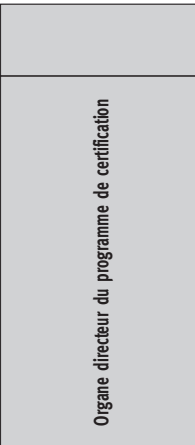 } & \multirow[b]{2}{*}{ 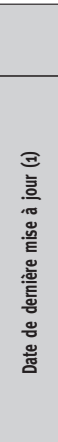 } & \multirow[b]{2}{*}{ 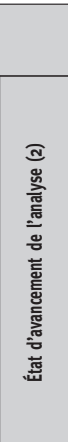 } & \multirow[b]{2}{*}{ 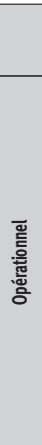 } & \multirow[b]{2}{*}{ 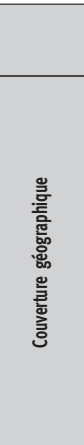 } & \multicolumn{2}{|c|}{\begin{tabular}{|c|}
$\begin{array}{c}\text { Affiliations } \\
(3)\end{array}$ \\
\end{tabular}} & \multicolumn{5}{|c|}{$\begin{array}{l}\text { Non discriminatoire } \\
(4)\end{array}$} & \multicolumn{2}{|c|}{$\begin{array}{l}\text { Indépendant } \\
\text { et impartial } \\
\text { (5) }\end{array}$} & \multicolumn{3}{|c|}{$\begin{array}{l}\text { Accréditation } \\
\text { (6) }\end{array}$} \\
\hline & & & & & 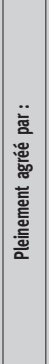 & 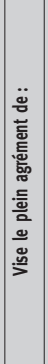 & 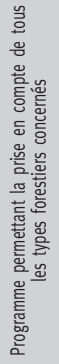 & 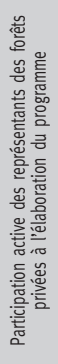 & 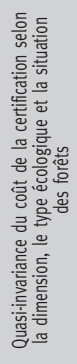 & 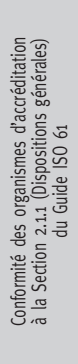 & 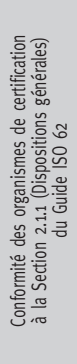 & 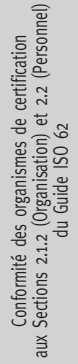 & 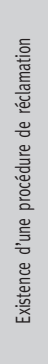 & 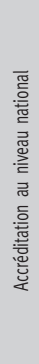 & 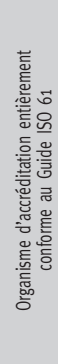 & 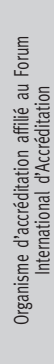 \\
\hline $\begin{array}{l}\text { AF\&PA Sustainable } \\
\text { Forestry Initiative (SFI) }\end{array}$ & 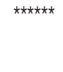 & $\begin{array}{l}\text { Projet } \\
\text { amendé }\end{array}$ & Oui & $\begin{array}{c}\begin{array}{c}\text { USA } \\
\text { (\& partie } \\
\text { du Canada) }\end{array} \\
\end{array}$ & & & ++ & ++ & ++ & ++ & ++ & ++ & ++ & ++ & ++ & ++ \\
\hline $\begin{array}{l}\text { CFV (Consejo Boliviano } \\
\text { para la Certificación } \\
\text { Forestal Voluntaria) }\end{array}$ & 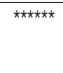 & $\begin{array}{l}\text { Avant- } \\
\text { projet }\end{array}$ & Oui & Bolivie & FSC & & ++ & $?$ & $?$ & na & na & na & na & 0 & na & na \\
\hline $\begin{array}{l}\text { Finnish Forest } \\
\text { Certification Council }\end{array}$ & 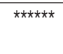 & \begin{tabular}{|r} 
Projet \\
amendé
\end{tabular} & Oui & Finlande & PEFC & & ++ & ++ & ++ & ++ & ++ & ++ & ++ & ++ & ++ & ++ \\
\hline $\begin{array}{l}\text { Foundation of the } \\
\text { Peoples of the South } \\
\text { Pacific, PNG }\end{array}$ & 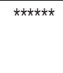 & $\begin{array}{l}\begin{array}{r}\text { Projet } \\
\text { amendé }\end{array} \\
\end{array}$ & Oui & $\begin{array}{l}\text { Papouasie } \\
\text { Nouvelle } \\
\text { Guinée }\end{array}$ & & FSC & ++ & + & $?$ & na & na & na & na & 0 & na & na \\
\hline $\begin{array}{l}\text { Lembaga Ekolabel } \\
\text { Indonesia (LEI) }\end{array}$ & 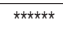 & \begin{tabular}{|l|} 
Projet \\
amendé
\end{tabular} & Oui & Indonésie & & FSC & + & + & $?$ & + & + & + & + & + & + & $?$ \\
\hline Living Forests & 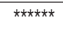 & $\begin{array}{l}\text { Avant- } \\
\text { projet }\end{array}$ & Oui & Norvège & PEFC & & ++ & ++ & $?$ & ++ & ++ & ++ & ++ & ++ & ++ & ++ \\
\hline LRF Skogsagama & 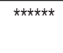 & $\begin{array}{l}\begin{array}{r}\text { Projet } \\
\text { amendé }\end{array} \\
\end{array}$ & Oui & Suède & PEFC & & ++ & ++ & ++ & ++ & ++ & ++ & ++ & ++ & ++ & ++ \\
\hline PEFC Austria & 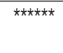 & $\begin{array}{l}\text { Projet } \\
\text { amendé }\end{array}$ & Oui & Autriche & PEFC & & ++ & ++ & ++ & ++ & ++ & ++ & ++ & ++ & ++ & ++ \\
\hline PEFC Germany & 4/10/00 & \begin{tabular}{|l} 
Projet \\
amendé
\end{tabular} & Oui & Allemagne & PEFC & & ++ & ++ & ++ & ++ & ++ & ++ & ++ & ++ & ++ & ++ \\
\hline $\begin{array}{l}\text { PEFC Switzerland and HWK- } \\
\text { Zertifizierungsstelle }\end{array}$ & 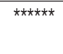 & $\begin{array}{l}\begin{array}{r}\text { Projet } \\
\text { amendé }\end{array} \\
\end{array}$ & Oui & Suisse & & PEFC & ++ & ++ & $?$ & ++ & ++ & ++ & ++ & ++ & ++ & ++ \\
\hline $\begin{array}{l}\text { Standards Council } \\
\text { of Canada }\end{array}$ & 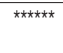 & $\begin{array}{l}\text { Avant- } \\
\text { projet }\end{array}$ & Oui & Canada & & & ++ & + & $?$ & ++ & ++ & ++ & ++ & ++ & ++ & ++ \\
\hline Swedish FSC Council & 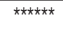 & $\begin{array}{l}\text { Avant- } \\
\text { projet }\end{array}$ & Oui & Suède & FSC & & ++ & + & $?$ & na & na & na & na & 0 & na & na \\
\hline $\begin{array}{l}\text { UK Woodland Assurance } \\
\text { Scheme Steering Group }\end{array}$ & $1 / 09 / 00$ & $\begin{array}{r}\begin{array}{r}\text { Projet } \\
\text { amendé }\end{array} \\
\end{array}$ & Oui & $\begin{array}{c}\text { Royaume- } \\
\text { Uni }\end{array}$ & FSC & & ++ & ++ & na & na & na & na & na & na & na & na \\
\hline $\begin{array}{l}\text { Associacao Brasileira de } \\
\text { Normas Tecnicas }\end{array}$ & 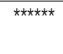 & $\begin{array}{l}\text { Avant- } \\
\text { projet }\end{array}$ & Non & Brésil & & & ++ & ++ & $?$ & 0 & ++ & ++ & ++ & 0 & 0 & 0 \\
\hline $\begin{array}{l}\text { CEF (Certificacion } \\
\text { Espanola Forestal) }\end{array}$ & 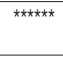 & \begin{tabular}{|l|} 
Avant- \\
projet
\end{tabular} & Non & Espagne & & PEFC & ++ & + & $?$ & + & $?$ & $?$ & $?$ & + & + & + \\
\hline $\begin{array}{l}\text { Conselho Da Fileira } \\
\text { Florestal Portuguesa } \\
\end{array}$ & 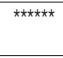 & \begin{tabular}{|l|} 
Projet \\
amendé
\end{tabular} & Non & Portugal & & PEFC & ++ & ++ & $?$ & ++ & ++ & ++ & ++ & ++ & ++ & + \\
\hline $\begin{array}{l}\text { Council of the National } \\
\text { Certification Centre - Czech }\end{array}$ & 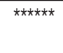 & \begin{tabular}{|l} 
Projet \\
amendé
\end{tabular} & Non & $\begin{array}{c}\text { Rép. } \\
\text { tchèque }\end{array}$ & & PEFC & ++ & ++ & ++ & ++ & ++ & ++ & ++ & ++ & ++ & ++ \\
\hline German FSC Contact Person & 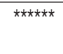 & $\begin{array}{l}\text { Avant- } \\
\text { projet }\end{array}$ & Non & Allemagne & FSC & & $?$ & 0 & $?$ & na & na & na & na & 0 & na & na \\
\hline $\begin{array}{l}\text { Ghana Forest Management } \\
\text { Certification Systems } \\
\text { Project }\end{array}$ & 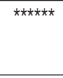 & $\begin{array}{l}\text { Avant- } \\
\text { projet }\end{array}$ & Non & Ghana & & & 0 & ++ & $?$ & $?$ & $?$ & $?$ & $?$ & $?$ & $?$ & $?$ \\
\hline $\begin{array}{l}\text { National Timber Certification } \\
\text { Council, Malaysia }\end{array}$ & $4 / 10 / 00$ & $\begin{array}{l}\begin{array}{r}\text { Projet } \\
\text { amendé }\end{array} \\
\end{array}$ & Non & Malaisie & & & ++ & ++ & + & + & ++ & ++ & ++ & + & + & + \\
\hline PEFC Council of Latvia & 3/10/00 & $\begin{array}{l}\begin{array}{l}\text { Projet } \\
\text { amendé }\end{array} \\
\end{array}$ & Non & Lettonie & & PEFC & + & + & ++ & ++ & ++ & ++ & ++ & ++ & ++ & 0 \\
\hline PEFC Denmark & $1 / 03 / 00$ & $\begin{array}{l}\text { Avant- } \\
\text { projet }\end{array}$ & Non & Danemark & & PEFC & ++ & + & $?$ & 0 & ++ & ++ & ++ & 0 & 0 & 0 \\
\hline PEFC France & $1 / 03 / 00$ & $\begin{array}{l}\text { Avant- } \\
\text { projet }\end{array}$ & Non & France & & PEFC & ++ & $?$ & $?$ & ++ & ++ & ++ & ++ & ++ & ++ & ++ \\
\hline $\begin{array}{l}\text { Sociadad para al Manego } \\
\text { Forestal Sustainable, A.C. }\end{array}$ & 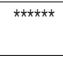 & $\begin{array}{l}\text { Avant- } \\
\text { projet }\end{array}$ & Non & Mexique & & FSC & ++ & $?$ & $?$ & na & na & na & na & 0 & na & na \\
\hline Woodnet asbl & $1 / 03 / 00$ & \begin{tabular}{|l} 
Projet \\
amendé
\end{tabular} & Non & Belgique & & PEFC & ++ & + & ++ & ++ & + & + & $?$ & ++ & ++ & ++ \\
\hline
\end{tabular}

Symboles : ++ Parfaitement conforme à l'indicateur ; + Partiellement conforme à l'indicateur; o Non-conforme à l'indicateur ; ? Données insuffisantes pour évaluer la conformité vis-à-vis de l'indicateur; 


\section{Systèmes nationaux de Certification des Forêts}

\begin{tabular}{|c|c|c|c|c|c|c|c|c|}
\hline \multicolumn{2}{|c|}{$\begin{array}{l}\text { Normes sur } \\
\text { les résultats } \\
(7)\end{array}$} & \begin{tabular}{|l} 
Normes sur \\
la gestion \\
(8)
\end{tabular} & \multicolumn{2}{|c|}{$\begin{array}{l}\text { Politique forestière } \\
\text { et réglementation } \\
(9)\end{array}$} & \multicolumn{4}{|c|}{$\begin{array}{l}\text { Action de recherche } \\
\text { de consensus } \\
\text { (10) }\end{array}$} \\
\hline 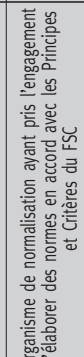 & 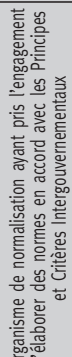 & 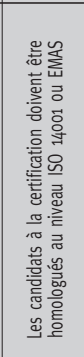 & 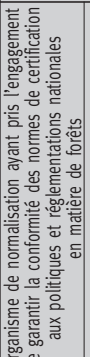 & 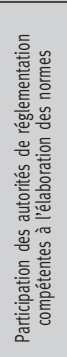 & 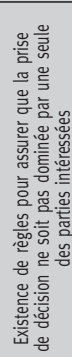 & 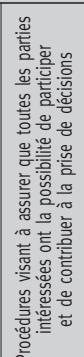 & 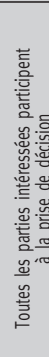 & 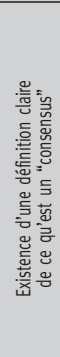 \\
\hline
\end{tabular}
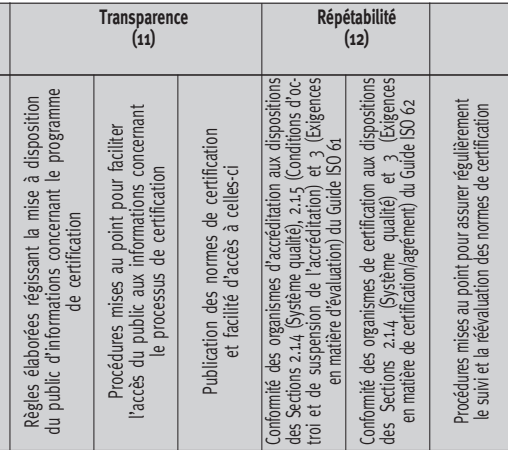

$\underset{(13)}{\text { Évolutif }}$

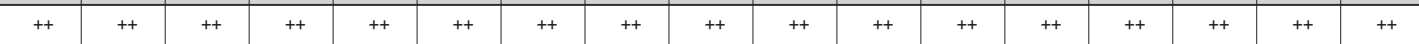

\begin{tabular}{|c|c|c|c|c|c|c|c|c|c|c|c|c|c|c|c|c|}
\hline++ & 0 & & $+t$ & + & $+t$ & + & t & $+t$ & na & & $+t$ & & & t+ & & ? \\
\hline++ & ++ & + & ++ & + & ++ & ++ & ++ & ++ & ++ & ++ & ++ & ++ & ++ & ++ & + & + \\
\hline++ & 0 & 0 & ++ & ++ & ++ & + & + & ++ & na & na & + & na & na & ++ & na & 0 \\
\hline++ & ++ & + & ++ & ++ & ++ & ++ & ++ & 0 & + & + & ++ & + & + & $?$ & ? & ++ \\
\hline++ & ++ & ++ & ++ & ++ & ++ & ++ & ++ & ++ & ++ & ++ & ++ & ++ & ++ & ++ & ++ & ? \\
\hline 0 & ++ & ++ & ++ & ++ & ++ & ++ & + & + & ++ & ++ & ++ & ++ & ++ & ++ & ++ & ++ \\
\hline
\end{tabular}

\begin{tabular}{|c|c|c|c|c|c|c|c|c|c|c|c|c|c|c|c|c|}
\hline 0 & ++ & 0 & ++ & ++ & ++ & ++ & + & ++ & ++ & ++ & ++ & ++ & ++ & ++ & ++ & ++ \\
\hline++ & ++ & + & ++ & ++ & ++ & ++ & + & ++ & ++ & ++ & ++ & ++ & ++ & ++ & ++ & ++ \\
\hline 0 & ++ & ++ & ++ & ++ & ++ & ++ & 0 & ++ & ++ & ++ & ++ & ++ & ++ & ++ & ++ & ? \\
\hline++ & ++ & 0 & ++ & $?$ & ++ & ++ & 0 & ++ & $\mathrm{na}$ & na & ++ & na & na & ++ & na & ? \\
\hline++ & ++ & + & ++ & ++ & ++ & ++ & ++ & ++ & na & na & ++ & na & na & ++ & na & na \\
\hline++ & ++ & 0 & ++ & ++ & ++ & ++ & ++ & ++ & ? & ? & ++ & 0 & ++ & ++ & ? & ? \\
\hline 0 & ++ & $?$ & ? & $?$ & ++ & + & + & ++ & ? & ? & $?$ & + & ? & $?$ & ? & ? \\
\hline
\end{tabular}

\begin{tabular}{|c|c|c|c|c|c|c|c|c|c|c|c|c|c|c|c|c|}
\hline 0 & ++ & + & ++ & ++ & ++ & ++ & + & ++ & ++ & ++ & $?$ & ++ & ++ & $?$ & ++ & $?$ \\
\hline++ & ++ & + & ++ & ++ & ++ & ++ & + & ++ & ++ & ++ & ++ & ++ & ++ & ++ & ++ & + \\
\hline++ & $?$ & 0 & $?$ & $?$ & ++ & $?$ & $?$ & ++ & na & na & ++ & na & na & + & na & $?$ \\
\hline++ & ++ & ++ & ++ & ++ & ++ & ++ & ++ & ++ & $?$ & $?$ & ++ & $?$ & $?$ & $?$ & $?$ & $?$ \\
\hline++ & ++ & $?$ & ++ & ++ & + & ++ & ++ & + & + & + & ++ & + & ++ & + & + & ++ \\
\hline+ & ++ & ++ & ++ & ++ & ++ & ++ & ++ & ++ & ++ & ++ & ++ & ++ & ++ & ++ & ++ & ++ \\
\hline ? & $?$ & $?$ & $?$ & ++ & $?$ & $?$ & + & $?$ & ++ & + & $?$ & 0 & + & $?$ & $?$ & $?$ \\
\hline 0 & ++ & ++ & ++ & $?$ & ++ & ++ & 0 & ++ & ++ & ++ & ++ & ++ & ++ & ++ & ++ & $?$ \\
\hline 0 & ++ & + & + & ++ & ++ & ++ & ++ & $?$ & + & + & $?$ & ++ & + & + & + & $?$ \\
\hline
\end{tabular}

na Indicateur inadapté au programme de certification. 


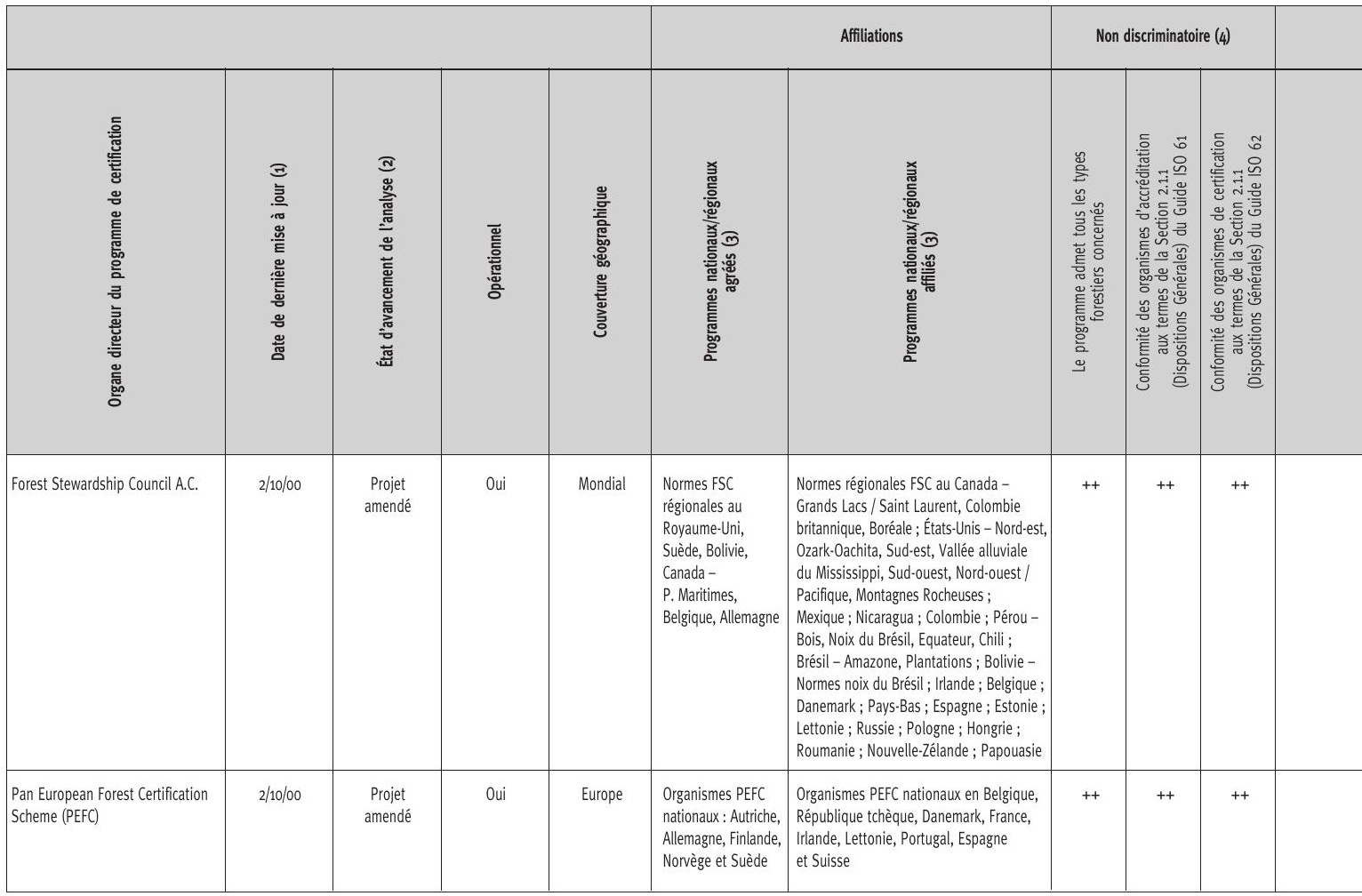

Symboles : ++ Parfaitement conforme à l'indicateur ; + Partiellement conforme à l'indicateur ; o Non-conforme à l'indicateur ; ? Données insuffisantes pour évaluer la conformité vis-à-vis de l'indicateur ;

\section{Actualisation de la matrice CEPI}

Une première version de la matrice de comparaison a été publiée début 2000, à une époque où de nombreux systèmes étaient encore en gestation ou au début de leur développement.

La CEPI a donc lancé, dans le courant de l'année 2000, une actualisation de son document, et c'est cette version "à jour" que nous publions ici.

Ce document donne donc la plus large vision possible des différents systèmes en cours de fonctionnement ou en phase d'installation, fin 2000.

Chacun peut alors établir son propre jugement, sur des bases aussi objectives que possible.

\section{ÉVOLUTIONS PRÉVUES POUR LA MATRICE DE COMPARAISON CEPI}

La CEPI a voulu créer cet outil qui jusqu'alors manquait, mais ne se considère pas pour autant propriétaire de la chose, qui a vocation à être utilisée et validée par tous. Par ailleurs, elle a bien conscience que la matrice peut et doit encore être améliorée. 
Systèmes internationaux de Certification des Forêts

\begin{tabular}{|c|c|c|c|c|c|c|c|c|c|c|c|c|c|c|c|c|}
\hline \multicolumn{2}{|c|}{$\begin{array}{l}\text { Indépendant et } \\
\text { impartial (5) }\end{array}$} & \multicolumn{3}{|c|}{$\begin{array}{c}\text { Procédures } \\
\text { d'accréditation (6) }\end{array}$} & \multicolumn{4}{|c|}{$\begin{array}{l}\text { Action de recherche } \\
\text { de consensus (10) }\end{array}$} & \multicolumn{2}{|c|}{ Transparence (11) } & \multicolumn{2}{|c|}{ Répétabilité (12) } & \multicolumn{2}{|c|}{ Évolutif (13) } & \multicolumn{2}{|c|}{$\begin{array}{l}\text { Étiquetage des } \\
\text { produits }(14)\end{array}$} \\
\hline 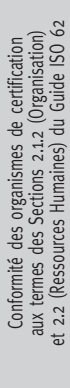 & 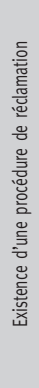 & 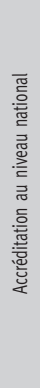 & 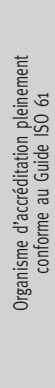 & 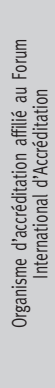 & 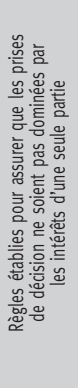 & 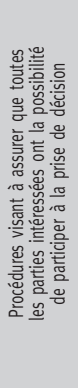 & 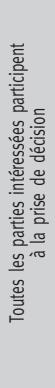 & 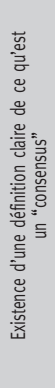 & 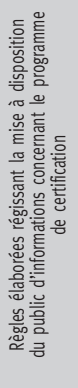 & 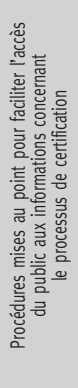 & 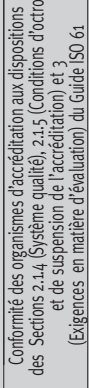 & 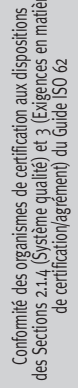 & 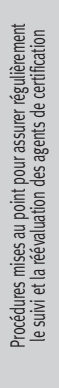 & 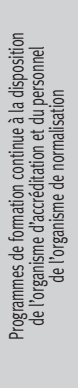 & 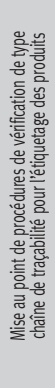 & 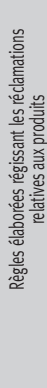 \\
\hline++ & ++ & na & ++ & na & ++ & ++ & + & ++ & ++ & ++ & ++ & ++ & ++ & ++ & ++ & ++ \\
\hline++ & ++ & ++ & ++ & ++ & ++ & ++ & + & ++ & ++ & ++ & ++ & ++ & ++ & ++ & ++ & ++ \\
\hline
\end{tabular}

na Indicateur inadapté au programme de certification.

En conséquence, les évolutions prévues sont :

- Réviser la liste des "caractéristiques attendues", et donc des critères et indicateurs qui leur sont liés, de façon à faire entrer dans le champ de l'examen des points de vue complémentaires. Ce travail sera proposé à l'ensemble des parties intéressées, avec comme objectif d'avoir terminé avant la fin du printemps, de façon à pouvoir relancer une actualisation des informations de la matrice.

- Publication de la version $n^{\circ} 3$ de la matrice, enrichie des points introduits par le travail cidessus, et mise à jour par circulation de nouveaux questionnaires.

- Ultérieurement : transmettre la gestion de la matrice à un organe indépendant.

\section{CONCLUSIONS}

L'apparition de nombreux systèmes a certes pu être dénoncée comme un facteur de confusion, mais dans une phase de "création" elle est néanmoins légitime, et même saine puisqu'elle permet à différentes visions de s'exprimer, et puisqu'elle stimule certainement la créativité et la 
recherche des meilleures solutions. À titre de comparaison, que ce serait-il passé s’il n'y avait eu qu'un seul constructeur automobile au monde ? Sans doute rien de bon pour le progrès...

Admettons donc que différents systèmes existent, et même sans doute que de nouveaux se créent encore, s'enrichissent mutuellement et apportent progressivement les meilleures réponses aux questions que pose la société.

Avec la matrice CEPI, nous disposerons désormais d'un outil fiable et actualisé pour comparer ces systèmes, et même sans doute pour les stimuler.

Mais au-delà, cet outil doit permettre d'avancer significativement vers la reconnaissance mutuelle.

\section{CERTIFICATION : CRÊATION D'UN OUTIL DE COMPARAISON DES DIFFÉRENTS SYSTĖmES EXISTANT DE PAR LE MONDE [Aésumé]}

Patrick OLLIVIER

Groupe La Rochette

2, rue Louis-David

F-75116 PARIS

La Confédération européenne des Industries du Papier (CEPI) a pris l'initiative de construire une matrice objective de comparaison des différents systèmes de certification présents sur le marché, sur la base de quelques caractéristiques de bon sens dont la liste a été établie par des experts en concertation avec les parties concernées. Une telle matrice permet non seulement de comparer les systèmes entre eux mais, sans doute aussi, de les stimuler voire de faciliter d'éventuelles discussions en vue d'une reconnaissance mutuelle.

\section{CERTIFICATION - THE ESTRBLISHMENT OF A TOOL FOR COMPARING THE DIFFERENT SYSTEMS WORLDUIDE [Abstract]}

The Confederation of European Paper Industries (CEPI) has taken the initiative of drafting a comparative matrix of the various certification systems available on the basis of a number of common sense indicators, the list of which was established by experts in cooperation with the interested parties. Not only does this matrix enable systems to be compared between themselves, it will no doubt furthermore provide stimulus and foster discussions for the purpose of reciprocal recognition. 